\title{
Contenido de materia orgánica en suelos de sistemas silvopastoriles establecidos en el Chaco Central paraguayo
}

\section{Organic matter content in soils of silvopastoral systems established in the Paraguayan Central Chaco}

\author{
Maura Isabel Díaz Lezcano ${ }^{*}$ (D) Cynthia Carolina Gamarra Lezcano ${ }^{1}$ (D), \\ Susana Ruiz Díaz ${ }^{1}$ (D) Mirtha Vera de Ortíz ${ }^{1}$ (D) \\ ${ }^{1}$ Universidad Nacional de Asunción, Facultad de Ciencias Agrarias. San \\ Lorenzo, Paraguay.
}

Autor de correspondencia: maura.diaz@agr.una.py

Resumen: El sistema silvopastoril se caracteriza por asociación de ganadería, árboles y pastura, la interacción entre estos componentes aporta beneficios tanto al suelo, como a la pastura, al ganado y a los árboles y permite un aumento en la productividad y la diversificación de la producción. Para conocer la influencia del componente arbóreo del sistema silvopastoril sobre el contenido de materia orgánica del suelo se llevó a cabo un estudio exploratorio en propiedades privadas ubicadas en los Departamento de Boquerón y Presidente Hayes del Chaco Central paraguayo. Se instalaron 8 parcelas permanentes de 1 ha cada una en las que se midieron y registraron todos los individuos de algarrobo (Prosopis spp), se identificaron y extrajeron muestras de pastura de subparcelas de $1 \mathrm{~m}^{2}$ que se ubicaron bajo la copa de los árboles y fuera de la copa para comparar la biomasa de la pastura, se extrajeron muestras de suelo bajo las condiciones mencionadas y a dos profundidades, de 0 a $10 \mathrm{~cm}$ y de 10 a $30 \mathrm{~cm}$, para comparar el contenido de materia orgánica, carbono y nitrógeno. El componente arbóreo estaba integrado por algarrobo blanco (Prosopis alba) y algarrobo negro (Prosopis nigra) asociados a pastura implantada, Gatton panic (Panicum máximum cv Gatton panic) pasto Pangola (Digitaria decumbens) y pasto Buffel (Cenchrus ciliaris) mayoritariamente. No se registraron diferencias significativas en el contenido de materia orgánica, carbono y nitrógeno entre condiciones de sol y de sombra, pero sí al variar la profundidad. Bajo las condiciones del 
presente estudio no se registró una influencia significativa del componente arbóreo sobre el contenido de materia orgánica.

Palabras clave: producción, algarrobo, suelos.

Abstract: The silvopastoral system is characterized by association of livestock, trees and pasture, the interaction between these components provides benefits to the soil, pasture, livestock and trees and allows an increase in productivity and diversification of production. In order to know the influence of the arboreal component of the silvopastoral system on the organic matter content of the soil, an exploratory study was carried out on private properties located in the Boquerón and Presidente Hayes Departments of the Paraguayan Central Chaco. Eight permanent plots of 1 ha each were installed in which all the carob trees (Prosopis spp) were measured and recorded, pasture samples were identified and extracted from subplots of $1 \mathrm{~m}^{2}$ that were located under the tree canopy and outside In order to compare the biomass of the pasture, the soil samples were extracted under the mentioned conditions and at two depths, from 0 to $10 \mathrm{~cm}$ and from 10 to $30 \mathrm{~cm}$, to compare the content of organic matter, carbon and nitrogen. The tree component was composed of white carob (Prosopis alba) and black carob (Prosopis nigra) associated with implanted pasture, Gatton panic (Panicum maximum cv Gatton panic), Pangola grass (Digitaria decumbens) and Buffel grass (Cenchrus ciliaris) mostly. There were no significant differences in the organic matter content, carbon and nitrogen between sun and shade conditions, but they did vary with depth. Under the conditions of the present study, there was no significant influence of the tree component on the content of organic matter.

Keywords: production, algarrobo, soils.

\section{INTRODUCCION}

En el Chaco paraguayo se consolidó el modelo ganadero semi intensivo. Debido a las condiciones edafoclimáticas es importante un manejo cuidadoso de recursos para lograr el aumento de la productividad; acciones como la implementación de sistemas silvopastoriles pueden ser muy beneficiosos(1).

Dentro del sistema silvopastoril, árboles, pasturas y animales de producción se combinan y manejan de manera racional e integral para lograr mejorar a mediano o largo plazo la productividad, la rentabilidad y la sustentabilidad de la explotación, además aportan condiciones que permiten el bienestar animal (sombra, protección contra vientos y heladas, disminución de 
temperatura y evapotranspiración), así como protección al suelo contra la erosión y la compactación, mejora su fertilidad a través del ciclaje y bombeo de nutrientes y acumulación de materia orgánica(2,3).

La materia orgánica está constituida por restos de animales y plantas (provenientes de hojas caídas, troncos muertos, raíces de árboles o hierbas y residuos de cosecha) en varios estados de descomposición y cumple importantes funciones, además es un indicador de calidad de los suelos pues condiciona sus propiedades físicas, químicas y biológicas ${ }^{(4,5)}$.

La importancia de este trabajo radica en que en el Chaco paraguayo las experiencias de beneficios del aprovechamiento de esta especie en sistemas silvopastoriles son escasas y aisladas. Tuvo como objetivo general analizar el contenido de materia orgánica en suelos de sistemas silvopastoriles asociados a Prosopis spp. del Chaco Central paraguayo y como objetivos específicos: caracterizar la composición arbórea y de la pastura dentro del sistema, evaluar el efecto de dos condiciones de insolación sobre la biomasa de la pastura y comparar el contenido de materia orgánica.

\section{MATERIALES Y MÉTODOS}

\section{Localización de la investigación}

El área de estudio está situada en los Distritos Pirizal, Buena Vista y Tte. Primero Manuel Irala Fernández del Departamento de Presidente Hayes, y Filadelfia y Loma Plata del Departamento de Boquerón de la Región Occidental del Paraguay, ubicada entre los paralelos $20^{\circ} 05^{\prime}$ y $23^{\circ} 48^{\prime}$ Latitud Sur y $62^{\circ} 40^{\prime}$ y $59^{\circ} 20^{\prime}$ Longitud Oeste de Greenwich, se encuentra a $420 \mathrm{~km}$ de la ciudad de Asunción. Limita al noreste con Alto Paraguay, al sur con la Región Oriental del país y al Suroeste con la Republica Argentina, teniendo como límite natural al Río Pilcomayo, que baña la región sur del departamento con afluentes no navegables pero importantes para el riego de suelos. Constituye un área de campos abiertos y vegetación espinosa y de tunas(6).

En la primera quincena de diciembre del 2015 se instalaron ocho parcelas permanentes de 1 ha, en distintas propiedades privadas ubicadas en los Departamentos de Presidente Hayes y Boquerón en las que se realizó un censo de algarrobos, extracción de muestras de pastura y muestras de suelo. La ubicación de las parcelas fue registrada mediante georreferenciaciones que pueden ser apreciadas en la Figura 1. 
Rev. Soc. cient. Parag. 2020;25(2):131-143

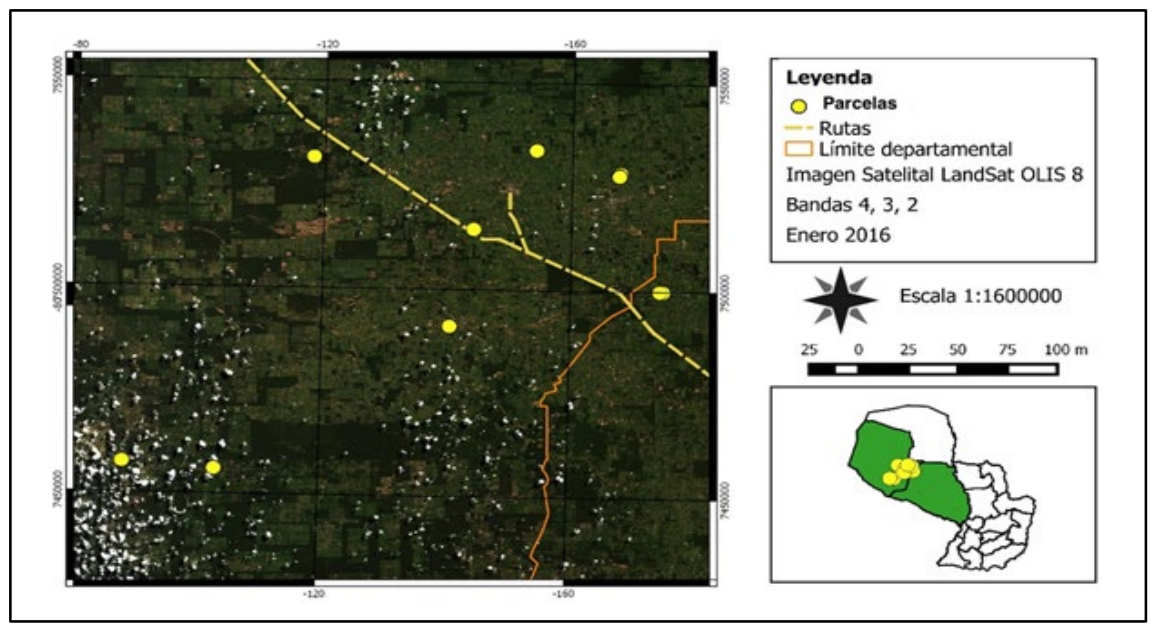

Figura1. Ubicación de las parcelas silvopastoriles.

Primeramente, se caracterizó el área de estudio considerando principalmente el distanciamiento entre los individuos arbóreos. Dentro de cada parcela se identificaron todas las especies arbóreas y de pastura presentes y se midieron y registraron todos los individuos de algarrobo, que fueron codificados con una chapa metálica.

Las variables medidas fueron:

- DAC (Diámetro a la altura del cuello): diámetro medido desde el suelo hasta el cuello de la planta $(\mathrm{cm})$.

- DAP (Diámetro a la altura del pecho): diámetro del árbol a los 1,30m de altura $(\mathrm{cm})$.

- Altura total: altura del árbol desde el suelo hasta el ápice (m).

- Biomasa total: peso de la materia orgánica que existe por encima y debajo del suelo (t.ha ${ }^{-1}$ ) (Ecuación alométrica propuesta por Sato et al. ${ }^{(7)}$ para el Chaco Seco $)=0,2733^{*}\left(h^{*} D^{2}\right)^{0,837}$

Por otra parte, para la extracción de muestras de pastura, se establecieron 8 subparcelas de $1 \mathrm{~m} \times 1 \mathrm{~m}$ dentro de cada parcela de 1 ha, estas subparcelas se distribuyeron al azar: 4 bajo influencia directa de la copa de algarrobos y 4 subparcelas en zonas fuera de la influencia directa de la copa del algarrobo. Las muestras fueron trasladadas al Laboratorio de Tecnología de la Madera de la Facultad de Ciencias Agrarias (FCA) de la Universidad Nacional de 
Rev. Soc. cient. Parag. 2020;25(2):131-143

Asunción (UNA), donde fueron secadas en estufa a $65^{\circ} \mathrm{C}$ hasta alcanzar peso constante y se obtuvo el peso seco equivalente a la biomasa.

Las variables medidas fueron:

- Peso seco: peso de la pastura luego de haber sido secada a horno (g).

- Biomasa: masa total de la pastura, se representa mediante su peso seco $(\mathrm{g})$.

Para la determinación de carbono orgánico en el suelo, en cada parcela de 1 ha se extrajeron cuatro muestras compuestas, constituida cada una de cuatro muestras simples extraídas aleatoriamente a dos profundidades, de 0 a $10 \mathrm{~cm}$ y de 10 a $30 \mathrm{~cm}$, bajo dos condiciones de insolación: bajo la influencia y fuera de la influencia de la copa de los algarrobos. Estas muestras fueron analizadas en el laboratorio del Área de Suelos y Ordenamiento Territorial de la Facultad de Ciencias Agrarias de la Universidad Nacional de Asunción donde se determinó el contenido de carbono orgánico a través del método Walkley Black $^{(8)}$, para poder calcular el contenido de materia orgánica, nitrógeno total aplicando fórmulas propuestas por la literatura a partir de los resultados del análisis.

A partir del contenido de carbono orgánico (C.0 \%), se calculó su contenido en toneladas por hectárea utilizando la siguiente formula:

$$
\text { C.O. (t.ha-1): (V* Da* C.O (\%)/100)/1000 }
$$

El volumen (V) de suelo se calculó multiplicando la superficie de la parcela por la profundidad de muestreo, la densidad aparente (Da) considerada fue de $1243 \mathrm{~kg} / \mathrm{m}^{3}$ para las muestras obtenidas de 0 a $10 \mathrm{~cm}$ y $1225 \mathrm{~kg} / \mathrm{m}^{3}$ para las muestras obtenidas de 10 a $30 \mathrm{~cm}$, los valores de volumen y densidad aparente se utilizaron además para determinar el contenido de materia orgánica y nitrógeno total en toneladas.

En cuanto al contenido de materia orgánica en el suelo, los valores fueron calculados aplicando la siguiente fórmula ${ }^{(9)}$.

$$
\text { M.O. (\%): C.O (\%)*1,72 }
$$

Por otra parte, el contenido de nitrógeno total se calculó considerando que el $5 \%$ de la materia orgánica está compuesto de nitrógeno( ${ }^{(4)}$ :

Nt: M.O(\%)*0,05 
Para el análisis de los datos se utilizó estadística descriptiva: y también se aplicó estadística comparativa por el método de parcelas no apareadas t de Student a un nivel de confianza del $95 \%$ debido a que las parcelas no se encontraban en condiciones homogéneas. Para comparar biomasa de pasturas bajo influencia directa de la copa del algarrobo y fuera de la influencia directa de ésta, contenido de materia orgánica bajo ambas condiciones de insolación y a dos profundidades, de 0-10 cm y de 10 a $30 \mathrm{~cm}$.

\section{RESULTADOS Y DISCUSION}

\section{Caracterización del área de estudio}

Considerando algunas excepciones, por medio de los mapas diseñados, se pudo notar un distanciamiento adecuado entre los árboles para permitir el ingreso de ganado y la siembra de la pastura, así como también el ingreso de las maquinarias para el trabajo de campo y el buen desarrollo de las copas de los árboles, el cual contaba con 9 metros de radio en promedio entre los algarrobos. El arreglo espacial de los árboles en un sistema silvopastoril consiste en una separación de 4 a 5 metros para facilitar el acceso y desplazamiento de los animales, también es necesario que el distanciamiento permita el acceso de maquinarias para tareas como preparación del suelo(10).

El distanciamiento también se relaciona con la cobertura de copas. La competencia por luz es el principal factor que determina la producción de forrajeras dado que el sombreamiento de las pasturas aumenta a medida que crecen los árboles y que la producción máxima de pastizales en zonas con déficit hídrico se alcanza con una cobertura arbórea del 50\%(11). Además, desde el punto de vista económico, el efecto de la sombra incrementa la producción de leche dentro de un rango de $10 \%$ al $20 \%$ en comparación a potreros sin árboles(12).

En todas las parcelas estudiadas se registraron individuos del género Prosopis, presentando de esta manera una frecuencia del 100\%. La capacidad de las especies del género Prosopis de tolerar sequía y condiciones edáficas adversas, como la salinidad y alcalinidad, así como su adaptación a la herbivoría, son las principales razones de su posición dominante en la vegetación leñosa de zonas áridas y semiáridas de América(13).

En 8 parcelas se observaron componentes forestales diferentes a los algarrobos. Las especies del género Prosopis son componentes importantes en el hábitat de otros organismos, generando heterogeneidad espacial, que 
modifican la distribución espacial de especies de los estratos arbustivos y herbáceos ${ }^{(13)}$.

Todos los individuos presentes en el área de estudio eran procedentes de regeneración natural, en ningún caso los algarrobos fueron implantados en los potreros. La presencia de árboles en los potreros es el resultado de la regeneración natural, dando lugar al ramoneo de forma alterna durante el ciclo anual. Los sistemas silvopastoriles facilitan la producción ganadera de forma integral y alivian la presión sobre las áreas boscosas, aumentado directa o indirectamente la productividad de la parcela(14).

\section{Componente arbóreo}

El componente arbóreo estaba integrado por algarrobo blanco (Prosopis alba) y algarrobo negro (Prosopis nigra). De los algarrobos registrados (247 individuos en total), 130 individuos (53\%) correspondían a la categoría de regeneración, con un $\mathrm{DAP}<10 \mathrm{~cm}$, y los 115 individuos restantes (47\%) tenían en promedio $16 \mathrm{~cm}$ de DAP, dentro de cada parcela se midieron en promedio 31 individuos.

Permitir el establecimiento de regeneraciones de algarrobos (Prosopis alba, Prosopis nigra o Prosopis kuntzei) con una densidad de 20 hasta 50 árboles por hectárea en pasturas degradadas puede traer beneficios ecológicos, pues la mayor acumulación de materia orgánica bajo sus copas contribuye a un mayor rendimiento de las pasturas para el ganado(15,16).

La biomasa aérea incluye todo lo que se encuentre sobre el suelo, como fuste, ramas y hojas, y la biomasa total incluye además de lo citado las raíces(17). En ese sentido, la biomasa del componente arbóreo del sistema fue de 2 tha-1 ${ }^{-1}$ en promedio, valor superior al estimado por Brítez ${ }^{(18)}$, que fue de 0,07 t.ha $^{-1}$ en una parcela de $2000 \mathrm{~m}^{2}$ bajo manejo silvopastoril con un alto grado de mortandad de árboles, ubicada en la misma ecorregión.

\section{Biomasa de pastura}

Los valores de biomasa de las pasturas varían según su exposición a la insolación, esto referido a la cobertura de copa de los árboles de algarrobo dentro del sistema silvopastoril.

Las especies de pastura que se encontraron con mayor frecuencia en las parcelas fueron Gatton panic (Panicum máximum cv. Gatton) Pangola (Digitaria decumbens) y Buffel (Cenchrus ciliaris), en menor proporción se 
registró la presencia de pasto Estrella (Cynodon nlemfuensis) y Urochloa (Urochloa mosambisensis)

Se presentaron diferencias significativas entre las muestras extraídas bajo la copa del algarrobo y las que se encontraban fuera de la influencia de la copa. En este sentido, la biomasa de la pastura que se encuentra fuera de la proyección de copa del algarrobo es en promedio de 0,46 t.ha $^{-1}$, variando entre 1,36 t. ha-1 y 0,2 t.ha- ${ }^{-1}$ en cambio, la media de la biomasa de la pastura bajo la copa equivale a 1,65 tha $^{-1}$ en promedio, variando entre 0,27 tha $\mathrm{a}^{-1} \mathrm{y}$ 1,64 tha ${ }^{-1}$. La Figura 2 presenta los valores promedios bajo ambas condiciones.

A su vez, Valle et al.(19) presentaron valores mayores $\left(3,5\right.$ t.ha-1 $\left.^{-1}\right)$ de biomasa en pasto Buffel de 3,5 meses de edad, asociadas a Gliricida sepium, árbol perteneciente a la familia de las leguminosas, en una parcela de $500 \mathrm{~m}^{2}$ ubicada en Miacatlán, México en condiciones similares al área de estudio de la presente investigación.

Por otro lado, Ibrahim et al.(20,21) expusieron que la pastura de la variedad Panicum maximum presentó mayor contenido de biomasa $\left(2,98\right.$ t.ha-1 $\left.^{-1}\right)$ al estar asociadas a árboles de Erithrina poeppigiana comparadas a 2,07 t.ha ${ }^{-1}$ de biomasa producida por la pastura cuando no está asociada a esta especie leñosa, esto podría atribuirse al efecto que ejercen las leguminosas sobre la fertilidad del suelo.

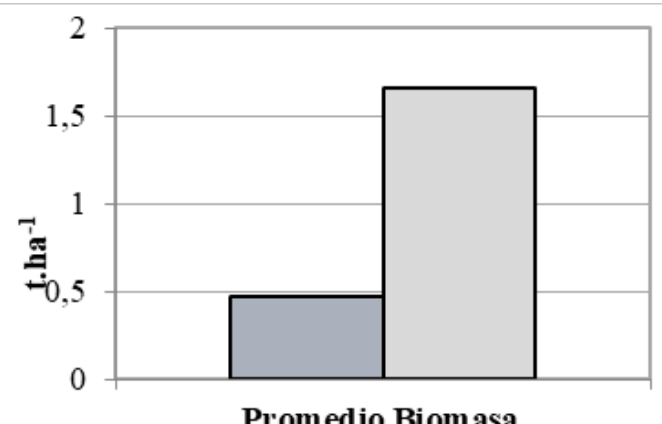

口Bajo sol

口Bajo sombra

Figura 2. Biomasa de pastura en promedio bajo dos condiciones de insolación. Nivel de confianza del 95\% Chaco Central. Diciembre 2015. 
Casado y Cavalieri(22) en cambio indicaron que en un sistema silvopastoril ubicado al sudoeste del Chaco argentino, bajo el dosel de Prosopis alba la biomasa de Gatton panic fue de 7,4 tha- ${ }^{-1} \mathrm{y}$ entre copas el valor fue de 6,7 tha1 .

\section{Materia orgánica, carbono y nitrógeno en suelos}

El contenido de materia orgánica del suelo no presentó diferencias significativas entre condiciones de sol y sombra, pero las diferencias fueron significativas entre las diferentes profundidades de muestreo, cuyos valores se presentan en la Figura 3.

En cuanto al contenido porcentual de materia orgánica, valores cercanos fueron expuestos por Glaztle ${ }^{(15,16)}$ quien expuso que el contenido de materia orgánica bajo la copa del algarrobo fue 3,3\% y descendió a 2,4\% en zonas despejadas, sin cobertura de algarrobo en un estudio realizado en el Chaco Central paraguayo.

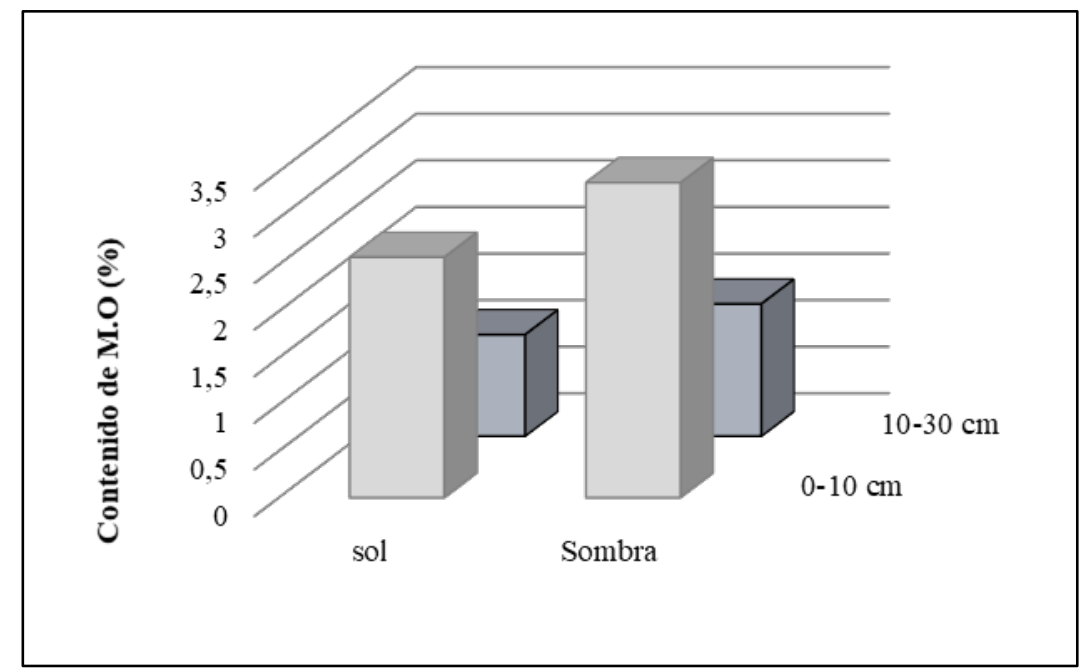

Figura 3. Promedio del contenido de materia orgánica en porcentaje en sistemas silvopastoriles del Chaco Central, 2015.

En la materia orgánica existen muchos elementos químicos importantes de entre los cuales son componentes funcionales y estructurales el carbono y el nitrógeno.

Las gramíneas utilizadas en la producción animal tropical generalmente son de metabolismo $\mathrm{C}_{4}$, lo cual les da mayor capacidad de integrarse en la materia 
orgánica de las plantas y éstas al ser consumidas por los animales regresa al suelo como heces y orina. Además, parte de las plantas se hacen senescentes y son incorporadas directamente al suelo(23).

El suelo es un gran reservorio de carbono, su concentración presenta altas variaciones incluso en áreas de tamaño pequeño debido a la heterogeneidad de los suelos, condiciones climáticas y elementos geomórficos(17), asimismo, Thompson y Throe ${ }^{(24)}$ refieren que la materia orgánica cubre la superficie del suelo, luego se descompone y se mezcla e incorpora a los 5-15 cm del suelo mineral gracias a la acción de la mesofauna que allí habita.

En total el contenido de carbono orgánico en el suelo bajo la influencia directa de la copa del algarrobo en los primeros $30 \mathrm{~cm}$ del suelo fue de 44,85 t.ha-1 $^{-1} \mathrm{y}$ fuera de la influencia fue de 34,03 t. ha-1 considerando una densidad aparente del suelo de $1,24 \mathrm{~g} / \mathrm{cm}^{3}$ y $1,25 \mathrm{~g} / \mathrm{cm}^{3}$ para las profundidades de $0-10 \mathrm{~cm}$ y 10 $30 \mathrm{~cm}$ respectivamente. Lok et al. ${ }^{(25)}$ reportan valores de carbono orgánico similares en un sistema silvopastoril con Panicum maximum y Leucaena leucocephala de 8 años, con un contenido de carbono orgánico de 38,8 t.ha ${ }^{-1}$ en los primeros $35 \mathrm{~cm}$ del suelo.

El contenido de Nitrógeno total en los primeros $10 \mathrm{~cm}$ de suelo fuera de la influencia de la copa del algarrobo fue de 1,6 tha ${ }^{-1} \mathrm{y}$ bajo la influencia de la copa fue de 2,03 t.ha-1y 1,34 t.ha-1 y 1,75 tha- ${ }^{-1}$ de 10 a $30 \mathrm{~cm}$ de profundidad bajo sol y sombra respectivamente.

Silberman et al.(26) expusieron un contenido de nitrógeno similar en los primeros $15 \mathrm{~cm}$ del suelo con cobertura de Gatton panic $\left(0,828 \mathrm{t} \cdot \mathrm{ha}^{-1}\right)$, y un valor superior $(4,14$ tha-1) en sistema silvopastoril basado en la asociación de Mistol (Ziziphus mistol) (22 a 27 árboles/ha) con Gatton panic, en un estudio realizado en Santiago del Estero, Chaco árido de Argentina.

\section{CONCLUSIÓN}

El contenido de materia orgánica, como los de carbono y nitrógeno, no presentaron diferencias significativas al comparar los niveles de insolación, sin embargo, al comparar a diferentes profundidades se observaron diferencias significativas. 
Rev. Soc. cient. Parag. 2020;25(2):131-143

Bajo las condiciones del presente estudio no se registró una influencia significativa del componente arbóreo sobre el contenido de materia orgánica en el suelo.

\section{AGRADECIMIENTOS}

Esta investigación se realizó en el marco del proyecto "Manejo Sustentable de Bosques en el Ecosistema Transfronterizo del Chaco" financiado por el Programa de las Naciones Unidas para el Desarrollo (PNUD).

\section{REFERENCIAS BIBLIOGRAFICAS}

1. Bazoberry 0. Chaco Boliviano Paraguayo: Desafío en perspectiva transfronteriza [internet] 2002. [fecha de consulta: Octubre 2015]. 1518 pp. Disponible en:

https://es.scribd.com/document/100927670/Chaco-bolivianoparaguayo-Desafios-en-perspectiva-transfronteriza

2. Martín G, Agüero S. Sistema Silvopastoril: una estrategia de producción para ecosistemas del NOA. [internet] 2009. Disponible en: http://www.produccionanimal.com.ar/produccion_y_manejo_pasturas/manejo\%20silvopastoril /106-noa.pdf

3. Nair PKR. An introduction to agroforestry. [internet] 1993. (Consultado 9 de septiembre de 2015) Disponible en: http://www.worldagroforestry.org/Units/Library/Books/PDFs/32_An_i ntroduction_to_agroforestry.pdf? $n=161$.

4. Plaster EJ. La ciencia del suelo y su manejo. Madrid, España: Paraninfo; 2000.

5. POSAF (Programa Socioambiental y Desarrollo Forestal). Establecimiento y manejo de sistemas silvopastoriles. Managua, NI, Gobierno de Nicaragua, Ministerio del Ambiente y de los Recursos Naturales. 2005; 56 p.

6. DGEEC (Dirección General de Estadística, Encuestas y Censos, PY). Atlas Censal del Paraguay. Fernando de la Mora, Paraguay; 2002.

7. Sato T, Saito M, Ramírez D, Molas L, Toriyama J, Monda Y, Kiyono Y, Herebia E, Dubie N, E. Duré E, Ramírez Ortega J, Vera de Ortiz M. Development of allometric equation for tree biomass in forest ecosystem in Paraguay. JARQ 49 (3), 281 - 291 [internet] 2015. (Consultado 19 de noviembre de 2016) Disponible en: http://www.jircas.affrc.go.jp

8. Tedesco MJ, Gianello C, Bissani CA, Bohnen H, Volkweiss, SJ. Análises do solo, plantas e outros materiais. 2da ed. Porto Alegre, Brazil: Universidade Federal do Rio Grande do Sul; 1995.

9. Porta J, López -Acevedo M, Poch RM. Edafología: Uso y protección de suelos. 3era ed. Madrid, España: Mundi-Prensa; 2014. 
10. POSAF (Programa Socioambiental y Desarrollo Forestal). Establecimiento y manejo de sistemas silvopastoriles. Managua, NI, Gobierno de Nicaragua, Ministerio del Ambiente y de los Recursos Naturales. 56 p; 2005.

11. Carranza C, Ledesma M. Bases para el manejo de sistemas silvopastoriles. Congreso Forestal Mundial. Buenos Aires, Argentina. 9 p; 2009.

12. Villanueva C, Ibrahim M, Cassasola F, Sepúlveda C. Sistemas silvopastoriles: una herramienta de adaptación al cambio climático de las fincas ganaderas en América Central. In Sepúlveda, C; Ibrahim, M. Políticas y sistemas de incentivo para el fomento y adopción de buenas prácticas agrícolas. Turrialba, Costa Rica: CATIE. 292 p. (Serie técnica); 2009.

13.Villagra, P. Aspectos ecológicos de los algarrobales argentinos. Multequina 9(2):35-51. [INTERNET] 2015. (Consultado 30 set 2015) Disponible en: http://www.cricyt.edu.ar/multequina/indice/pdf/09_02/9_2_3.pdf

14. Ramírez N, Pérez M, Ferguson B, Jiménez G. Caracterización del sistema silvopastoril en la depresión central de Chiapas. Avances en Investigación Agropecuaria. 16(2):7-22. [INTERNET] 2012. (Consultado 28 de septiembre de 16). Disponible en: http://www.ucol.mx/revaia/portal/pdf/2012/mayo/1.pdf

15. Glatzle A. Compendio para el manejo de pastura en el Chaco. Asunción, Paraguay: Editorial El Lector; 1999.

16. Glatzle A. Sistemas productivos en el Chaco Central Paraguayo. [INTERNET] 2006. (Consultado 17 de octubre de 2016) Disponible en: http://www.chaconet.com.py/inttas/projects/pdf/a_glatzle_sistemas_pr oductivos.pdf.

17. Yepes A, Navarrete D, Duque A, Phillips JF, Cabrera KR, Álvarez E, García ME, Ordoñez MF (eds.). Protocolo para la estimación de nacional y subnacional de biomasa-carbono en Colombia. Bogotá, Colombia: IDEAM; 2011.

18. Brítez, CN. Estimación del contenido de carbono en un sistema integrado de producción en el Departamento de Presidente Hayes, Chaco Paraguayo. San Lorenzo, Paraguay: Tesis de grado. Facultad de Ciencias Agrarias, Universidad Nacional de Asunción; 2015.

19. Valle JL, Palma JM, Sagines GL. Biomasa y composición nutricional de la asociación Cenchrus ciliaris-Gliricida sepium al establecimiento . [internet] 2004. (Consultado 04 de diciembre de 2016). Disponible en: http://www.ucol.mx/revaia/anteriores/anteriores/2004/VOL.2/Bioma sa $\% 20 y \% 20$ composicion $\% 20$ nutricional $\% 20 \mathrm{de} \% 20 \mathrm{la} \% 20$ asociacion $\%$ 20Cenchrus\%20.pdf.

20. Ibrahim M, Pezo D. Sistema silvopastoril. 2da ed. Turrialba, Costa Rica: CATIE/GTZ; 1999.

21. Ibrahim M, Schlonvoigt A, Camargo J, Souza M. Multi-strata silvopastoral systems for increasing productivity and conservation of natural resources 
Rev. Soc. cient. Parag. 2020;25(2):131-143

in Central America. [internet] 2001. (Consultado 17 de octubre de 2016) Disponible

en:

http://www.internationalgrasslands.org/files/igc/publications/2001/te ma18-1.pdf.

22. Casado MV, Cavalieri JM. Reconocimiento de especies forrajeras (Gatton panic). [internet] 2015. (Consultado 19 de noviembre de 2016) Disponible en: http://inta.gov.ar/documentos/Reconocimientode especies forrajeras gatton panic.pdf

23. Fisher MJ, Rao IM, Ayarza CE, Lascano CE, SanzJI, Thomas RJ, Vera RR. 1994. Carbon storage by introduced deep-rooted grasses in the South American savannas. [internet] 1994. (Consultado 29 de octubre de 2016) Nature. 371, 236-238.

24. Thompson LM, Troeh FR,. Los suelos y su fertilidad. 4ta ed. Barcelona, España: Editorial Reverté; 2013

25. Lok S, Fraga S, Noda A, García, M. Almacenamiento de carbono en el suelo de tres sistemas ganaderos tropicales en explotación de ganado vacuno. [internet] 2013. (Consultado 17 de octubre de 2016) Disponible en: www.redalyc.org/pdf/1930/193028545014.pdf.

26. Silbermann JE, Anriquez AL, Domínguez J, Kunts C; Albanesi, AS. La cobertura arbórea en un sistema silvopastoril del Chaco y su contribución diferencial al suelo. Revista Ciencia del Suelo. Vol 33(1). [internet] 2015. (Consultado 10 de noviembre de 2016) Disponible en: http://www.scielo.org.ar/scielo.php?script=sci_arttext\&pid=S185020672015000100003.

Díaz Lezcano MI, Gamarra Lezcano CC, Ruiz Díaz S, Vera de Ortíz M. Contenido de materia orgánica en suelos de sistemas silvopastoriles establecidos en el Chaco Central paraguayo. 